\title{
A espirometria na avaliação pré e pós-transplante de medula óssea*
}

\author{
Pre-operative and post-operative spirometry in bone marrow transplant patients
}

\author{
Eliane Viana Mancuzo', Walace Espada da Silva ${ }^{2}$, Nilton Alves de Rezende ${ }^{3}$
}

\begin{abstract}
Resumo
Objetivo: Analisar os resultados da espirometria de pacientes submetidos a transplante de medula óssea e verificar sua importância na detecção de complicações pulmonares e sua correlação com a evolução dos pacientes. Métodos: Foram analisados retrospectivamente os resultados da espirometria em 120 pacientes, maiores de doze anos, de ambos os sexos, e comparados com o tipo de transplante de medula óssea, doença de base, sorologia para citomegalovírus, fonte de células para o transplante, tabagismo, infecção pulmonar, doença pulmonar prévia, duração da doença hematológica, quimioterapia utilizada, regime de condicionamento, doença do enxerto contra o hospedeiro aguda e crônica e óbito. Resultados: Dezesseis pacientes apresentaram alterações da espirometria antes do transplante, sendo 5\% com obstrução pura, 5,8\% com restrição pura e 2,5\% com obstrução com redução da capacidade vital. Após o transplante 29 pacientes apresentaram alterações desses exames. A chance de alteração da espirometria foi maior nos pacientes com doença do enxerto contra o hospedeiro aguda $(p=0,02)$, idade menor que 30 anos $(p=0,02)$, sexo feminino $(p=0,02)$ e naqueles que receberam células tronco $(p=0,01)$. As presenças de doença pulmonar prévia e doença do enxerto contra o hospedeiro crônica associaram-se com aumento da mortalidade. Alterações prévias da espirometria não estiveram relacionadas com o óbito pós-transplante. Conclusão: As alterações detectadas na espirometria não foram capazes de predizer a ocorrência de complicações pulmonares e óbito pós-transplantes. Também não foram determinantes para a não realização do procedimento. A espirometria simples realizada na avaliação desses pacientes parece ter pouca importância prática.
\end{abstract}

Descritores: Espirometria; Transplante de medula óssea; Período pré-operatorio.

\begin{abstract}
Objective: To analyze the spirometry findings in patients undergoing bone marrow transplant, determining the importance of such findings in predicting postoperative pulmonary complications and looking for correlations with postoperative outcomes. Methods: The spirometry findings in 120 male and female patients, all above the age of 12, were evaluated retrospectively and compared in terms of the following parameters: the type of bone marrow transplant; the underlying disease; cytomegalovirus serology; source of the transplanted cells; smoking; pulmonary infection; history of lung disease; duration of the hematological disease; chemotherapy employed; conditioning regimen; acute or chronic rejection of the transplant; and post-operative mortality. Results: In the pre-operative spirometry, 16 patients (13.3\%) presented alterations: $6(5 \%)$ presented pure obstruction; 7 (5.8\%) presented pure restriction; and $3(2.5 \%)$ presented obstruction accompanied by a reduction in vital capacity. In the post-operative spirometry, 29 patients $(24.2 \%)$ presented alterations. The chance of presenting postoperative spirometry alterations was greater in patients presenting acute transplant rejection $(p=0.02)$, patients older than 30 ( $p=0.02)$, female patients $(p=0.02)$ and patients receiving stem cells $(p=0.01)$. Having a history of lung disease was found to be associated with greater mortality, as was suffering from chronic transplant rejection. No relationship was found between pre-operative spirometry alterations and post-operative mortality. Conclusion: In bone marrow transplant patients, the alterations found through pre-operative spirometry were not predictive of post-operative pulmonary complications or mortality. Nor were such alterations determinant of whether or not a given patient was a good candidate for bone marrow transplant. Simple spirometry seems to be of little practical importance in the evaluation of such patients.
\end{abstract}

Keywords: Spirometry; Bone marrow transplantation/adverse effects; Bone marrow transplantation/mortality.

\footnotetext{
* Trabalho realizado na Faculdade de Medicina da Universidade Federal de Minas Gerais - UFMG - Belo Horizonte (MG) Brasil.

1. Médica do Serviço de Pneumologia do Hospital das Clínicas da Universidade Federal de Minas Gerais - HC - UFMG - Belo Horizonte (MG) Brasil.

2. Acadêmico de Medicina da Universidade Federal de Minas Gerais - UFMG - Belo Horizonte (MG) Brasil.

3. Professor Adjunto do Departamento de Clínica Médica da Universidade Federal de Minas Gerais - UFMG - Belo Horizonte (MG) Brasil.

Endereço para correspondência: Professor Nilton Alves de Rezende. Departamento de Clínica Médica da Faculdade de Medicina da UFMG. Al. Alfredo Balena, 190, CEP 30130-100, Belo Horizonte, MG, Brasil. E-mail: narezende@terra.com.br

Recebido para publicação em 21/2/2006. Aprovado, após revisão, em 15/5/2006.
} 


\section{Introdução}

As complicações pulmonares constituem causa importante de morbidade e mortalidade após o transplante de medula óssea (TMO). ${ }^{(1)} A$ utilização dos testes de função pulmonar (TFP) na avaliação pré-operatória e no acompanhamento pós-operatório, além de identificar complicações não infecciosas pós-transplante, pode permitir a adoção de medidas preventivas e terapêuticas precoces em pacientes de risco. ${ }^{(2)}$

As principais alterações funcionais pulmonares verificadas após o TMO são: doenças pulmonares obstrutivas, que se caracterizam por redução do volume expiratório forçado do primeiro segundo $\left(V F_{1}\right)$ e redução da relação entre $V_{E F}$ e capacidade vital forçada (CVF) (a bronquiolite obliterante é a complicação pulmonar de pior prognóstico no TMO); doenças pulmonares restritivas, que são definidas como redução da capacidade pulmonar total (CPT) e da capacidade vital, com as relações $\mathrm{VEF}_{1} / \mathrm{CVF}$ e entre fluxo expiratório forçado entre 25\% e 75\% da CVF e CVF preservadas ou elevadas (os fatores mais comuns associados a esta condição após TMO são a irradiação torácica, a quimioterapia tóxica pulmonar, infecções, pneumonia idiopática e doença do enxerto contra o hospedeiro - GVHD); distúrbios ventilatórios obstrutivos com CVF reduzida (neste caso a CVF reduzida pode se dever apenas ao processo obstrutivo ou a processo restritivo associado, sendo necessário a medida da CPT para o seu diagnóstico); distúrbios combinados, quando se observa obstrução associada à restrição, medida através da CPT (no TMO podem ser encontrados pacientes com bronquiolite obliterante que evoluem com fibrose pulmonar associada); redução da difusão pulmonar de monóxido de carbono (DLCO), anormalidade funcional mais comum, ocorrendo em cerca de 50\% dos pacientes (os fatores de risco são os mesmos para a doença pulmonar restritiva)..$^{(1-8)}$

Desde a implantação do serviço de TMO no Hospital das Clínicas da Universidade Federal de Minas Gerais (HC-UFMG) em julho de 1995, a espirometria simples com prova broncodilatadora é realizada rotineiramente na avaliação da função pulmonar pré-transplante e no acompanhamento dos pacientes pós-transplante. Embora realizados há mais de dez anos, não se sabe exatamente a importância desses testes na detecção precoce e na adoção de medidas preventivas e/ou terapêuticas na abor- dagem das complicações pulmonares pós-TMO. Desta forma, a análise evolutiva dos pacientes submetidos ao TMO no HC-UFMG que realizaram espirometria antes e após o transplante pode ser importante e pode permitir a adoção de novos protocolos para a avaliação da função pulmonar nessa população.

\section{Métodos}

Trata-se de um estudo retrospectivo em que foram analisados os prontuários médicos de todos os pacientes submetidos a TMO no HC-UFMG no período de julho de 1995 a janeiro de 2004. Foram incluídos todos os pacientes maiores de doze anos, de ambos os sexos, em que se pôde obter os resultados da espirometria antes e pelo menos uma vez após o TMO.

As espirometrias foram realizadas no Laboratório de Função Pulmonar do HC-UFMG nos equipamentos Collins DS 11a With Universal Breathing Valve e Koko Spirometer 92494. As curvas volumetempo e fluxo-volume foram realizadas atendendo às normas preconizadas pelas Diretrizes para Testes de Função Pulmonar. ${ }^{(8)}$ Todas as curvas dos testes espirométricos foram revistas pelos autores deste trabalho.

Os resultados da espirometria (capacidade vital, CVF, $V_{E F} / C V F$ e relação entre fluxo expiratório forçado entre $25 \%$ e $75 \%$ da CVF e CVF) foram as variáveis de interesse principal. Os resultados dos exames foram classificados em: normal, obstrutivo, restritivo ou obstrutivo com redução da capacidade vital. ${ }^{(8)}$ Para esta classificação utilizou-se a tabela de referência de Pereira et al. ${ }^{(7)}$ em pacientes do sexo masculino maiores de 24 anos e do sexo feminino maiores de 20 anos. Para os demais pacientes foram considerados alterados os exames com valores abaixo de $80 \%$ do valor de referência normal. ${ }^{(9)}$ As variáveis independentes analisadas foram: sexo, idade, diagnóstico da doença de base, sorologia do receptor e doador para citomegalovírus, fonte de células para o TMO, tabagismo, quimioterapia utilizada pré-TMO, presença de doença pulmonar prévia, infecção pulmonar pré e pós-TMO, duração da doença hematológica, regime de condicionamento utilizado, uso de irradiação corporal total (ICT), ocorrência de GVHD aguda e GVHD crônica (em um dos seguintes sítios: pele, boca, olhos, fígado, intestino e pulmão) e óbito (caracterizado por morte em qualquer período após cem dias do transplante). 
Foi considerado como quimioterapia tóxica pulmonar o uso de pelo menos um dos seguintes medicamentos: hidroxiuréia, citosina arabinosídeo, daunoblastina, ciclofosfamida, bussulfam, bleomicina, metotrexate e ciclosporina. . $^{(1,10-13,19)}$

As informações coletadas foram digitadas em um banco de dados desenvolvido no programa Epi Info ${ }^{\mathrm{TM}}$, versão 6.04 (Center for Disease Control and Prevention - CDC, Atlanta, domínio público). As variáveis com valor de $p \leq 0,25$ na análise univariada (Qui-quadrado ou teste de Fisher) foram incluídas em um modelo de regressão logística, no qual se considerou que valores de $p<0,05$ indicavam, de forma independente, que apresentavam associação estatisticamente significativa.

0 projeto deste estudo foi aprovado pelo Comitê de Ética em Pesquisa da Universidade Federal de Minas Gerais (Parecer ETIC 43904, 17/11/2004).

\section{Resultados}

Dos 368 pacientes cujos prontuários foram analisados, 95 faleceram antes de 100 dias e, portanto, não fizeram a segunda espirometria, 19 tinham menos de doze anos na época do transplante e 134 não realizaram testes de função pulmonar após o transplante. Desta forma, 120 pacientes preencheram os critérios de inclusão e compuseram a casuística deste estudo. Tinham menos de 30 anos $43 \%$ dos pacientes, com distribuição semelhante entre os sexos. A taxa de mortalidade foi de 10\%. Quanto ao diagnóstico da doença de base, 50\% eram portadores de leucemia mielóide crônica. Vinte e quatro pacientes eram tabagistas $(20 \%)$ e 89 foram submetidos à quimioterapia préTMO (74\%). Infecção pulmonar antes do TMO foi observada em 3 pacientes (2,5\%). Sessenta pacientes tinham menos de um ano de evolução da doença de base (50\%). Nenhum paciente foi submetido a ICT.

Nesta casuística, todos os transplantes foram alogênicos. Cerca de um terço dos pacientes apresentaram GVHD aguda em pelo menos um sítio e dois terços apresentaram GVHD crônica. A infecção pulmonar pós-TMO ocorreu em 22,5\% dos pacientes. Mais de $90 \%$ dos pacientes e doadores eram soropositivos para citomegalovírus e fizeram uso de bussulfam e ciclofosfamida no regime de condicionamento (Tabela 1). Antes do transplante, 16 pacientes apresentaram alterações na espirometria $(13,3 \%)$ e 100 dias após o procedimento, este número foi de 29 pacientes $(24,2 \%)$ (Tabela 2$)$.
Tabela 1 - Características do transplante de medula óssea e complicações pós-transplantes.

\begin{tabular}{lrr}
\hline & $n$ & $\%$ \\
\hline Características & & \\
\hline Tipo de transplante & & \\
$\quad$ Alogênico & 120 & 100,0 \\
Profilaxia da GVHD & & \\
$\quad$ Ciclosporina + metotrexate & 119 & 99,2 \\
$\quad$ Outros* & 1 & 0,8 \\
Fonte de células & & \\
$\quad$ Medula óssea & 51 & 42,5 \\
$\quad$ Células tronco & 69 & 57,5 \\
Regime de condicionamento & & \\
$\quad$ Bussulfan + ciclofosfamida & 108 & 90,0 \\
$\quad$ Outros** & 12 & 10,0 \\
\hline Complicações pós-transplante & & \\
\hline GVHDa & & \\
$\quad$ Pulmão & 0 & 0,0 \\
$\quad$ Um ou mais sítios & 44 & 36,7 \\
GVHDc & & \\
$\quad$ Pulmão & 8 & 6,7 \\
$\quad$ Um ou mais sítios & 80 & 66,7 \\
Infecção pulmonar pós-transplante & & \\
$\quad$ Sim & 27 & 22,5 \\
Não & 93 & 77,5 \\
\hline
\end{tabular}

*Ciclosporina; ***Ciclofosfamida; GVHDa: doença do enxerto contra o hospedeiro aguda; e GVHDc: doença do enxerto contra o hospedeiro crônica.

$\mathrm{Na}$ análise univariada, os pacientes que apresentaram GVHD aguda em um ou mais sítios, idade igual ou inferior a 30 anos e sexo feminino tiveram maior chance de terem a espirometria alterada 100 dias após o transplante. Aqueles que receberam células tronco periféricas tiveram também uma tendência de associação estatística com esta alteração (Tabela 3).

Tabela 2 - Resultados da espirometria antes e após o transplante de medula óssea.

\begin{tabular}{|c|c|c|c|c|c|c|}
\hline & \multicolumn{2}{|c|}{ antes } & \multicolumn{2}{|c|}{100 dias } & \multicolumn{2}{|c|}{$\begin{array}{l}\text { Um ano } \\
\text { depois }\end{array}$} \\
\hline & $\mathrm{n}$ & $\%$ & $n$ & $\%$ & $\mathrm{n}$ & $\%$ \\
\hline Normal & 104 & 86,7 & 88 & 73,3 & 20 & 16,7 \\
\hline Obstrução & 6 & 5,0 & 10 & 8,3 & 8 & 6,7 \\
\hline Restrição & 7 & 5,8 & 12 & 10,0 & 0 & 0,0 \\
\hline $\begin{array}{l}\text { Obstrução com } \\
\text { redução da CVF }\end{array}$ & 3 & 2,5 & 7 & 5,8 & 6 & 5,0 \\
\hline Sem exames & 0 & 0,0 & 3 & 2,5 & 86 & 71,7 \\
\hline
\end{tabular}

CVF: capacidade vital forçada. 
Tabela 3 - Comparação entre os resultados da espirometria 100 dias após o transplante e variáveis independentes.

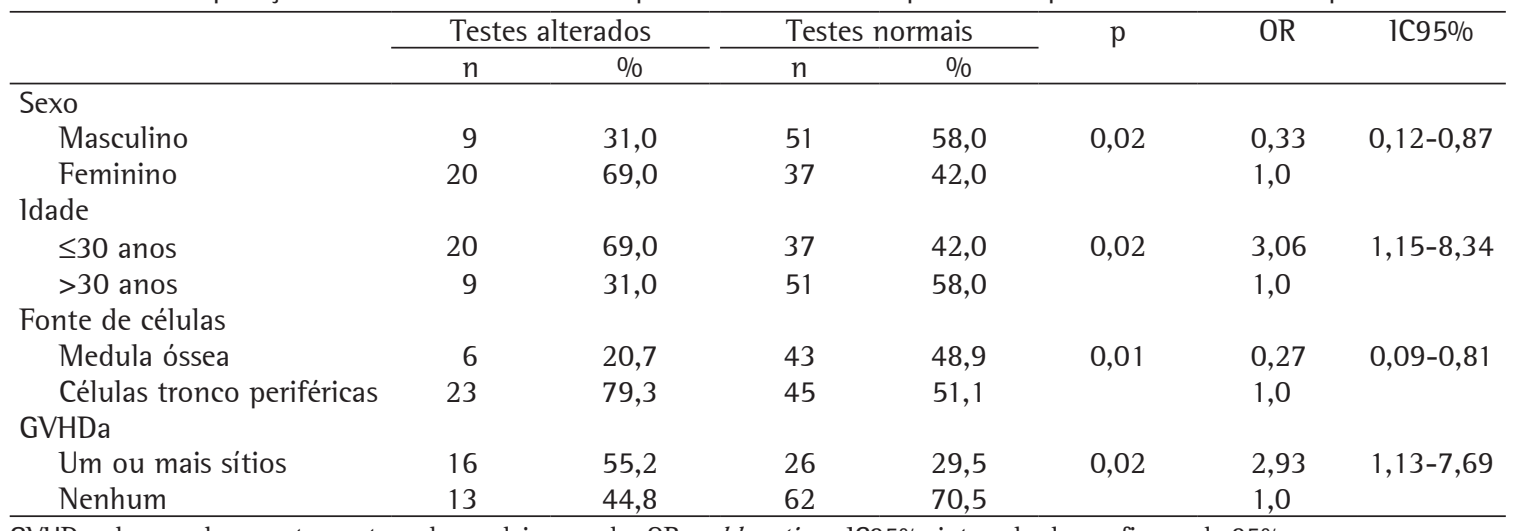

GVHDa: doença do enxerto contra o hospedeiro aguda; OR: odds ratio; e 1C95\%: intervalo de confiança de 95\%.

Os pacientes portadores de doença pulmonar prévia estiveram estatisticamente associados com mortalidade aumentada pós-transplante e aqueles que desenvolveram GVHD crônica em um ou mais sítios tiveram também uma tendência de associação com aumento da mortalidade. As demais variáveis independentes analisadas não mostraram significância estatística em relação à mortalidade (Tabela 4). Na análise multivariada, verificou-se que apenas os pacientes do sexo feminino, aqueles com idade igual ou menor que 30 anos e os que tiveram GVHD aguda mostraram maior chance de apresentarem espirometria alterada 100 dias após o TMO e a presença de doença pulmonar prévia foi a única que esteve associada ao óbito pós-transplante (Tabela 5).

\section{Discussão}

A literatura internacional, assim como diversos serviços de TMO, recomenda a realização de testes de função pulmonar antes e no acompanhamento pós-transplante. A argumentação para a realização destes testes é que os mesmos são importantes para o diagnóstico de complicações pulmonares não infecciosas pós-transplantes e para a adoção precoce de medidas terapêuticas e conseqüente aumento da sobrevida dos pacientes. ${ }^{(1,2,4,10)}$ Entretanto, este aspecto não pôde ser constatado em nosso trabalho. Deve ser ressaltado, todavia, que dos 368 pacientes submetidos ao TMO no HC-UFMG no período de estudo, apenas 120 foram analisados, o que pode ter comprometido os resultados desta pesquisa. Por outro lado, deve ser salientado que, em revisão sistemática da literatura, a maioria dos estudos é constituída por menos de 100 pacientes. ${ }^{(14)}$ Devem também ser salientadas as limitações de nosso estudo, que teve como fonte de dados a consulta retrospectiva em prontuários médicos e o fato de que apenas a espirometria foi avaliada, enquanto que os estudos mais importante registrados na literatura se referem à espirometria, avaliação da CPT e da DLCO..$^{(1,4,12,13-14,19,21)}$

Os distúrbios da função pulmonar no TMO são classicamente divididos em obstrutivos, restritivos e alterações da difusão pulmonar do monóxido de carbono, que é a alteração mais comumente observada. ${ }^{(1,4)}$ Os trabalhos não mencionam a obstrução associada à redução da capacidade vital (CVF). Em nosso estudo observamos que alguns pacientes, tanto antes como após o transplante, apresentaram distúrbio obstrutivo com CVF reduzida. Como a medida da CPT não foi realizada rotineiramente, não foi possivel analisar nestes pacientes se a CVF estava reduzida por restrição associada ou por aprisionamento aéreo. A medida da DLCO não é realizada rotineiramente no protocolo do Serviço de TMO do HC-UFMG, o que impediu sua análise.

A irradiação corporal total é um fator de risco importante para alterações dos TFP tanto antes como após o TMO..$^{(2,13,15-18)}$ Neste estudo nenhum paciente recebeu irradiação corporal total antes do transplante. Este fato pode ter diminuído a chance de alteração da função pulmonar tanto antes como após o transplante. Alguns autores relataram alterações também na DLCO e na CPT pós-transplantes em pacientes submetidos a irradiação corporal total. ${ }^{(18-19)}$

Nesta série de casos verificamos a persistência das alterações espirométricas detectadas antes do transplante e 100 dias após a realização do 
Tabela 4 - Risco de óbito entre as variáveis independentes.

\begin{tabular}{|c|c|c|c|c|c|c|c|}
\hline & \multicolumn{2}{|c|}{ Óbito } & \multicolumn{2}{|c|}{ Sobreviventes } & \multirow[t]{2}{*}{$p$} & \multirow[t]{2}{*}{ OR } & \multirow[t]{2}{*}{ 1C95\% } \\
\hline & $\mathrm{n}$ & $\%$ & $\mathrm{n}$ & $\%$ & & & \\
\hline \multicolumn{8}{|l|}{ Quimioterapia prévia } \\
\hline Sim & 10 & 83,3 & 81 & 75,0 & \multirow[t]{2}{*}{0,72} & \multirow[t]{2}{*}{1,67} & \multirow[t]{2}{*}{$0,31-11,9$} \\
\hline Não & 2 & 16,7 & 27 & 25,0 & & & \\
\hline \multicolumn{8}{|l|}{ Doença pulmonar prévia } \\
\hline Sim & 4 & 33,3 & 5 & 4,6 & \multirow[t]{2}{*}{$<0,01$} & 10,3 & \multirow[t]{2}{*}{$1,82-59,5$} \\
\hline Não & 8 & 66,7 & 103 & 95,4 & & 1,0 & \\
\hline \multicolumn{8}{|c|}{ Quimioterapia tóxica pulmonar } \\
\hline Não usou & 2 & 16,7 & 28 & 25,9 & \multirow{3}{*}{$\begin{array}{l}1,0 \\
0,25\end{array}$} & 1,0 & \multirow{3}{*}{$\begin{array}{l}0,20-10,0 \\
0,44-24,15\end{array}$} \\
\hline Hidréia & 5 & 41,7 & 56 & 51,9 & & 1,25 & \\
\hline Outras* & 5 & 41,7 & 24 & 22,2 & & 2,92 & \\
\hline \multicolumn{8}{|l|}{ GVHDa } \\
\hline Um ou mais sítios & 4 & 33,3 & 40 & 37,4 & \multirow[t]{2}{*}{1,0} & 0,84 & \multirow[t]{2}{*}{$0,19-3,38$} \\
\hline Nenhum & 8 & 66,7 & 67 & 62,6 & & 1,0 & \\
\hline \multicolumn{8}{|c|}{ Espirometria à admissão } \\
\hline Alterada & 2 & 16,7 & 14 & 13,0 & \multirow[t]{2}{*}{0,67} & 1,34 & \multirow[t]{2}{*}{$0,00-7,79$} \\
\hline Normal & 10 & 83,3 & 94 & 87,0 & & 1,0 & \\
\hline \multicolumn{8}{|c|}{ Espirometria cem dias depois } \\
\hline Alterada & 4 & 33,3 & 25 & 23,8 & \multirow[t]{2}{*}{0,48} & 1,60 & \multirow[t]{2}{*}{$0,36-6,66$} \\
\hline Normal & 8 & 66,7 & 80 & 76,2 & & 1,0 & \\
\hline \multicolumn{8}{|l|}{ GVHDc } \\
\hline Um ou mais sítios & 11 & 91,7 & 69 & 63,9 & \multirow[t]{2}{*}{0,06} & 6,22 & \multirow[t]{2}{*}{$0,77-135,9$} \\
\hline Nenhum & 1 & 8,3 & 39 & 36,1 & & 1,0 & \\
\hline \multicolumn{8}{|c|}{ Infecção pulmonar pós-TMO } \\
\hline Sim & 5 & 41,7 & 22 & 20,4 & \multirow[t]{2}{*}{0,14} & 2,79 & \multirow[t]{2}{*}{$0,68-11,27$} \\
\hline Não & 7 & 58,3 & 86 & 79,6 & & 1,0 & \\
\hline
\end{tabular}

*Citosina arabinosídeo, daunoblastina; GVHDa: doença do enxerto contra o hospedeiro aguda; GVHDc: doença do enxerto contra o hospedeiro crônica; e TMO: transplante de medula óssea.

Tabela 5 - Modelo de regressão logística para espirometria alterada 100 dias após transplante e óbito.

\begin{tabular}{|c|c|c|c|c|}
\hline Variáveis & Coeficiente & OR & $1 \mathrm{C} 95 \%$ & $p$ \\
\hline \multicolumn{5}{|l|}{ Espirometria alterada } \\
\hline Sexo (feminino) & 1,2761 & 3,58 & $1,32-9,76$ & 0,013 \\
\hline ldade ( $\leq 30$ anos) & 1,4503 & 4,26 & $1,55-11,74$ & 0,005 \\
\hline Fonte de células (tronco periféricas) & 1,0425 & 2,84 & $0,97-8,29$ & 0,057 \\
\hline GVHDa & 1,2378 & 3,45 & $1,29-9,25$ & 0,014 \\
\hline \multicolumn{5}{|l|}{ Óbito } \\
\hline Doença pulmonar prévia & 2,505 & 12,25 & $2,36-63,57$ & 0,003 \\
\hline GVHDc & 2,071 & 7,93 & $0,89-71,02$ & 0,064 \\
\hline
\end{tabular}

GVHDa: doença do enxerto contra o hospedeiro aguda; GVHDc: doença do enxerto contra o hospedeiro crônica; OR: odds ratio; e 1C95\%: intervalo de confiança de 95\%.

mesmo. Estas alterações, entretanto, não estiveram relacionadas com as complicações pulmonares mais freqüentemente descritas na literatura, como bronquiolite obliterante, obstrução grave ao fluxo aéreo e infecção pós-transplante. ${ }^{(20-25)} \mathrm{Em}$ dois estudos recentes foram relatadas alterações funcionais pulmonares pré-transplantes relacionadas com complicações pós-transplantes. ${ }^{(4,24)}$ Nestes estudos foram observados que a relação $\mathrm{VEF}_{1} / \mathrm{CVF}$ menor que $80 \%$ esteve relacionada com risco duas vezes maior de obstrução ao fluxo aéreo pós-transplante. Este aspecto não foi observado em nosso estudo, à semelhança de outros autores que também não encontraram associação entre alterações funcionais pré-transplantes e complicações pós-transplantes. ${ }^{(26)}$

$\mathrm{Na}$ análise dos fatores de risco associados a alterações pulmonares pós-transplantes verificamos uma associação significativa destas alterações com a GVHD aguda. Este achado está de acordo com o que é descrito na literatura, que mostra uma freqüência 
maior de pneumonia intersticial em pacientes com GVHD aguda. ${ }^{(13,19)}$ Da mesma forma, outros autores relataram GVHD aguda e GVHD crônica como fatores de risco para alterações da espirometria pós-TMO. Entretanto, estes autores relataram que esta complicação ocorre predominantemente em pacientes com idade superior a 60 anos, ${ }^{(24,27)}$ ao contrário do nosso estudo, em que a idade inferior a 30 anos foi a que esteve mais freqüentemente relacionada com alterações funcionais pós-TMO. Em apenas um estudo anteriormente publicado foi demonstrado como único fator de risco associado a alteração dos TFP pós-transplante a idade acima de oito anos. ${ }^{(28)}$ Deve ser salientado que crianças muito jovens não conseguem realizar de forma satisfatória a espirometria. Como não incluímos pacientes com idade inferior a doze anos, este dado não pôde ser confrontado com os da literatura.

Os pacientes do sexo feminino apresentaram maior chance de alterações da espirometria póstransplante. Outros autores também encontraram associação entre alterações dos TFP e o sexo feminino. ${ }^{(29)}$ Entretanto, estes autores encontraram alterações somente da DLCO, parâmetro não analisado em nossa casuística.

Observamos em nossa análise uma tendência de associação entre alteração da espirometria póstransplante e pacientes que receberam células tronco periféricas como fonte de células para o transplante. Pacientes que recebem células tronco têm maior chance de $\mathrm{GVHD}^{(30)}$ e, portanto, alteração dos TFP. Entretanto, esta associação, até onde sabemos, não foi ainda descrita na literatura.

Apesar de haver vários relatos de toxicidade pulmonar por drogas e sua relação com alterações dos TFP, ${ }^{(2,12-13,19,21)}$ esta associação não pôde ser demonstrada neste trabalho. Deve ser ressaltado que muitos pacientes de nosso estudo não realizaram a espirometria em 100 dias pós-transplante, o que pode ter prejudicado a análise dos resultados.

Doze pacientes evoluíram para o óbito (10\%). Não encontramos associação significativa entre espirometria alterada pré ou pós-transplante com esta evolução. Esta constatação está de acordo com alguns autores que também não encontraram aumento de complicações pulmonares fatais. ${ }^{(3,12)}$ Entretanto, em um destes estudos, ${ }^{(12)}$ a DLCO menor que $80 \%$ e o gradiente alvéolo-arterial maior que $20 \mathrm{mmHg}$ estiveram associados com aumento da mortalidade. Estes mesmos autores, em outro estudo, observaram que distúrbio restritivo ou uma redução maior que 15\% na CPT após três meses de transplante estiveram associados com maior risco de mortalidade. ${ }^{(21)}$

A caracterização específica da doença pulmonar prévia não pôde ser definida, pois não constava no protocolo de atendimento da maioria dos pacientes. 0 fato de estes pacientes não terem espirometria alterada pode ser explicado, pelo menos em parte, pela ausência da realização da medida de volumes pulmonares absolutos e da difusão pulmonar do monóxido de carbono, que são métodos mais sensíveis para diagnosticar alterações funcionais pulmonares. Entretanto, a presença de doença pulmonar prévia esteve associada ao óbito póstransplante. Este aspecto reforça a necessidade de se analisar, de forma completa, as características funcionais pulmonares em pacientes candidatos ao TM0, já que foi relatada uma associação significativa entre presença de problemas pulmonares com aumento da mortalidade pós-TMO. ${ }^{(24)}$ Neste estudo foi relatada uma taxa de mortalidade de $9 \%$ em três anos, $12 \%$ em cinco anos e 18\% em dez anos nos pacientes com obstrução ao fluxo aéreo.

Apesar do amplo conhecimento das alterações funcionais pulmonares e das complicações pulmonares descritas em pacientes submetidos ao TMO, as alterações da espirometria pré-TMO não foram consideradas contra-indicação à realização do procedimento. A espirometria alterada antes do TMO não esteve relacionada com aumento de complicações pulmonares pós-transplante. A despeito do aumento das alterações espirométricas pós-TMO, não foi possível demonstrar que estas alterações modificaram a evolução dos pacientes. Deve ser ressaltado que neste estudo as alterações da DLCO, as mais freqüentemente encontradas no TM0, não foram analisadas. Assim, a espirometria, acompanhada de medidas da DLCO e da CPT, deve ser avaliada em trabalhos prospectivos para que estes testes possam efetivamente serem incorporados como exames importantes na avaliação pré e pós-transplante de medula óssea.

\section{Agradecimentos}

Agradecemos ao Conselho Nacional para o Desenvolvimento Científico e Tecnológico do Brasil e à Comissão de Aperfeiçoamento Permanente de Ensino Superior pelo auxílio para a realização deste estudo. 


\section{Referências}

1. Marras TK, Szalai JP, Chan CK, Lipton JH, Messner HA, Laupacis A. Pulmonary function abnormalities after allogeneic marrow transplantation: a systematic review and assessment of an existing predictive instrument. Bone Marrow Transplant. 2002;30(9):599-607.

2. Marras TK, Chan CK, Lipton JH, Messner HA, Szalai JP, Laupacis A. Long-term pulmonary function abnormalities and survival after allogenic marrow transplantation. Bone Marrow Transplant. 2004;33(5):509-17.

3. Ratanatharathorn V, Ayash L, Lazarus HM, Fu J, Uberti JP. Chronic graft-versus-host disease: clinical manifestation and therapy. Bone Marrow Transplant. 2001;28(2):121-9.

4. Chien JW, Madtes DK, Clark JG. Pulmonary function testing prior to hematopoietic stem cell transplantation. Bone Marrow Transplant. 2005;35(5):429-35.

5. Cerveri 1, Zoia MC, Fulgoni P, Corsico A, Casali L, Tinelli C, Zecca M, Giorgiani G, Locatelli F. Late pulmonary sequelae after childhood bone marrow transplantation. Thorax. 1999;54(2):131-5.

6. Nenadov Beck M, Meresse V, Hartmann 0, Gaultier C. Longterm pulmonary sequelae after autologous bone marrow transplantation in children without total body irradiation. Bone Marrow Transplant. 1995;16(6):771-5.

7. Pereira CAC, Barreto SP, Simões JG, Pereira FWL, Gerstler JG, Nakatani J. Valores de referência para espirometria em uma amostra da população brasileira adulta. J Pneumol. 1992;18(1):10-22.

8. Sociedade Brasileira de Pneumologia e Tisiologia. Diretrizes para testes de função pulmonar. J Pneumol. 2002;28(3):1-221.

9. Knudson RJ, Lebowitz MD, Hgolberg CJ, Burrows B. Changes in the normal maximal expiratory flow-volume curve with growth and aging. Am Rev Respir Dis. 1983;127(6):725-34.

10. Quintas-Cardama A, Perez-Encinas M, Gonzalez S, Bendana A, Bello JL. Hydroxyurea-induced acute interstitial pneumonitis in a patient with essential thrombocythemia. Ann Hematol. 1999;78(4):187-8.

11. Morgan M, Dodds A, Atkinson K, Szer J, Downs K, Biggs J. The toxicity of busulphan and cyclophosphamide as the preparative regimen for bone marrow transplantation. $\mathrm{Br} \mathrm{J}$ Haematol. 1991;77(4):529-34.

12. Crawford SW, Petersen FB. Long-term survival from respiratory failure after marrow transplantation for malignancy. Am Rev Respir Dis. 1992;145(3):510-4.

13. Sutedja TG, Apperley JF, Hughes JM, Aber VR, Kennedy HG, Nunn P, et al. Pulmonary function after bone marrow transplantation for chronic myeloid leukaemia. Thorax. 1988;43(3):163-9.

14. Mancuso EV, Rezende NA. [Pulmonary function testing in bone marrow transplantation: a systematic review] Rev Port Pneumol. 2006;12(1):61-9. Portuguese.

15. Ghalie R, Szidon JP, Thompson L, Nawas YN, Dolce A, Kaizer $H$. Evaluation of pulmonary complications after bone marrow transplantation: the role of pretransplant pulmonary function tests. Bone Marrow Transplant. 1992;10(4):359-65.

16. Little MT, Storb R. History of hematopoeitic stem-cell transplantation. Nat Rev Cancer. 2002;2(3):231-8.
17. Carlson K, Backlund L, Smedmyr B, Oberg G, Simonsson B. Pulmonary function and complications subsequent to autologous bone marrow transplantation. Bone Marrow Transplant. 1994;14(5):805-11.

18. Gore EM, Lawton CA, Ash RC, Lipchik RJ. Pulmonary function changes in long-term survivors of bone marrow transplantation. Int J Radiat Oncol Biol Phys. 1996;36(1):67-75.

19. Badier M, Guillot C, Delpierre S, Vanuxem P, Blaise D, Maraninchi D. Pulmonary function changes 100 days and one year after bone marrow transplantation. Bone Marrow Transplant. 1993;12(5):457-61.

20. Kotloff RM, Ahya VN, Crawford SW. Pulmonary complications of solid organ and hematopoietic stem cell transplantation. Am J Respir Crit Care Med. 2004;170(1):22-48.

21. Crawford SW, Pepe M, Lin D, Benedetti F, Deeg HJ. Abnormalities of pulmonary function tests after marrow transplantation predict nonrelapse mortality. Am J Respir Crit Care Med. 1995;152(2):690-5.

22. Clark JG, Hansen JA, Hertz MI, Parkman R, Jensen L, Peavy $\mathrm{HH}$. NHLBl workshop summary. Idiopathic pneumonia syndrome after bone marrow transplantation. Am Rev Respir Dis. 1993;147(6 Pt 1):1601-6.

23. Kantrow SP, Hackman RC, Boeckh M, Myerson D, Crawford SW. Idiopathic pneumonia syndrome: changing spectrum of lung injury after marrow transplantation. Transplantation. 1997;63(8):1079-86.

24. Chien JW, Martin PJ, Gooley TA, Flowers ME, Heckbert SR, Nichols WG, et al. Airflow obstruction after myeloablative allogeneic hematopoietic stem cell transplantation. Am J Respir Crit Care Med. 2003;168(2):208-14.

25. Santo Tomas LH, Loberiza FR Jr, Klein JP, Layde PM, Lipchik RJ, Rizzo JD, et al. Risk factors for bronchiolitis obliterans in allogeneic hematopoietic stem-cell transplantation for leukemia. Chest. 2005;128(1):153-61. Erratum in: Chest. 2006;129(1):216.

26. Schwarer AP, Hughes JM, Trotman-Dickenson B, Krausz T, Goldman JM. A chronic pulmonary syndrome associated with graft-versus-host disease after allogeneic marrow transplantation. Transplantation. 1992;54(6):1002-8.

27. Schultz KR, Green GJ, Wensley D, Sargent MA, Magee JF, Spinelli JJ, et al. Obstructive lung disease in children after allogeneic bone marrow transplantation. Blood. 1994;84(9):3212-20.

28. Leneveu H, Bremont F, Rubie H, Peyroulet MC, Broue A, Suc A, et al. Respiratory function in children undergoing bone marrow transplantation. Pediatr Pulmonol. 1999;28(1):31-8.

29. Nysom K, Holm K, Hesse B, Ulrik CS, Jacobsen N, Bisgaard $\mathrm{H}$, et al. Lung function after allogeneic bone marrow transplantation for leukaemia or lymphoma. Arch Dis Child. 1996;74(5):432-6.

30. Azevedo WM. Transplante alogênico de células-tronco periféricas mobilizadas por rhG-GSF, não manipuladas "in vitro", para tratamento de neoplasias hematológicas [tese]. Campinas: Faculdade de Ciências Médicas da Universidade Estadual de Campinas; 1995. 